\title{
A FUNCTIONAL INEQUALITY AND ITS RELATION TO CONVEXITY OF VECTOR- VALUED FUNCTIONS
}

\author{
IH-CHING HSU
}

DEDICATED TO PROFESSOR EINAR HILLE, FOR HIS 82ND BIRTHDAY

\begin{abstract}
With respect to a partial ordering «, the functional inequality $F(s)+t G(s) \ll F(s+t)$ arises naturally in the study of extending classical convex-function theory to vector-valued functions. The solution $F$ is strongly convex and has a Riemann type integral representation, even a Bochner type integral representation when the functional inequality is considered in a Banach lattice. The paper also proves the equivalence of strong and weak convexity in an ordered locally convex space whose positive cone is closed. As an application, an affirmative answer is given to an open question raised earlier by R. G. Kuller and the author.
\end{abstract}

1. Introduction. This paper illustrates some initial steps taken in the efforts of extending classical theory of convexity to vector-valued functions. We start out with the extension of the basic definitions. The strong convexity and weak convexity are defined for functions assuming values in ordered vector spaces. As suspected (or even expected), the possible equivalence of these two basic convexities depends on the availability of the Hahn-Banach Theorem. It is therefore interesting to find out that locally convex ordered topological vector spaces with closed cones of positive elements are those general structures in which the validity of the Hahn-Banach Theorem indeed leads to the equivalence of strong and weak convexity. We then study the integral representation of the vector-valued convex-function-solution to an inequality which evolves from the classical theory of convexity. We obtain the order-convergent Riemann type integral, and realize that, for norm convergent integrals, the least we should require is a partial ordering compatible with the norm topology. Banach lattices therefore become our choices. A Banach lattice $(V, \ll,\|\cdot\|)$ is a Banach space with a vector lattice structure such that $|x| \ll|y|$ implies $\|x\| \leqslant\|y\|$; here $x, y$ are vectors in $V$ and $|x|=x^{+}+x^{-}$. A Banach lattice $V$ also has a property: $V^{b}=V^{*}$ i.e., the space $V^{b}$ of all order-bounded linear functionals on $V$ is the same as the space $V^{*}$ of all norm continuous linear functionals on $V[1$, p. 368]. This striking property and the compatible

Presented to the Society, October 24, 1973; received by the editors July 16, 1974 and, in revised form, October 16, 1975.

AMS (MOS) subject classifications (1970). Primary 46A40; Secondary 46G10, 26 A51.

Key words and phrases. Weak convexity, strong convexity, Hahn-Banach Theorem, ordered locally convex space, positive cone, order-convergence, Banach lattice, order-bounded, strong Lebesgue measurable, Bochner integral, Riesz descomposition theorem.

(c) American Mathematical Society 1976 
partial ordering play important roles in our derivation of Bochner type integral representation for the convex-function-solution to the inequality when being considered in Banach lattices.

2. Equivalence of weak and strong convexity. Throughout this paper, let $\ll$ denote the partial ordering in an ordered vector space with $\theta$ as the zero vector; and let $(a, b)$ denote an open interval on the real line which has the usual ordering $\leqslant$.

Definition 1. Let $V$ be an ordered vector space. A function $F:(a, b) \rightarrow V$ is strongly convex on $(a, b)$ if

$$
F\left(\frac{1}{2} s+\frac{1}{2} t\right) \ll \frac{1}{2} F(s)+\frac{1}{2} F(t),
$$

whenever $s$ and $t$ are in $(a, b)$.

DEFINITION 2. Let $W$ be a locally convex ordered topological vector space. A function $F:(a, b) \rightarrow W$ is weakly convex on $(a, b)$ if, for each positive continuous linear functional $p$ on $V$,

$$
p\left[F\left(\frac{1}{2} s+\frac{1}{2} t\right)\right] \leqslant \frac{1}{2} p[F(s)]+\frac{1}{2} p[F(t)],
$$

whenever $s$ and $t$ are in $(a, b)$.

Clearly, strong convexity implies weak convexity. The converse is valid in ordered spaces with the property that, if $p(x) \geqslant 0$ for all positive continuous linear functionals $p$, then $x \gg \theta$. Basically this is the main concern of the following

Lemma. Let $V$ be an ordered locally convex space whose cone $C$ of positive elements is closed. Then $x \in C$ if and only if $p(x) \geqslant 0$ for all positive continuous linear functionals $p$ on $V$.

Proof. Suppose $x \notin C$. Then by a theorem in [2, p. 220], there exists a nontrivial continuous linear functional $q$ on $V$, and a real number $\alpha$ such that $q(x)<\alpha \leqslant q(y)$ for all $y$ in $C$. For arbitrary $y$ in $C$ and arbitrary positive integer $n, n y \in C$. Therefore $\alpha \leqslant q(n y)$ and $\alpha / n \leqslant q(y)$. It follows that $q(y) \geqslant 0$ for all $y$ in $C$. Meantime $q(x)<\alpha \leqslant q(\theta)=0$ since the zero vector $\theta$ is in $C$. Thus, if $x \notin C$, there exists a positive continuous linear functional $q$ such that $q(x)<0$. This proves the nontrivial part of the lemma.

As a direct consequence of the lemma, we state the following

THEOREM 1. Weak and strong convexity are equivalent in an ordered locally convex space whose cone of positive elements is closed.

An application of this theorem to Banach lattices, which are examples of ordered locally convex spaces with closed positive cone [3, p. 175], gives an affirmative answer to the open question raised in [4, p. 366].

3. A functional inequality. By putting together several results in $[5, \mathrm{pp} .9,94$, 95], a characterization of real-valued convex functions can be formulated as follows: A real-valued function $f$ is convex on $(a, b)$ if and only if there exists a function $g$ on $(a, b)$ such that $f(s)+\operatorname{tg}(s) \leqslant f(s+t)$ whenever $s$ and $s+t$ are in $(a, b)$.

Considering the above functional inequality in ordered vector spaces, the 
following theorem gives partial answers to two related open questions: (1) Can vector-valued convex functions be characterized by a similar functional inequality? (2) Can vector-valued convex functions be characterized by integral representations as being done for real-valued convex functions as in $[6, \mathrm{pp} .9,10]$ ?

THEOREM 2. In an ordered vector space $(V, \ll)$, consider the functional inequality

$$
F(s)+t G(s) \ll F(s+t), \quad s \text { and } s+t \text { in }(a, b),
$$

where $G:(a, b) \rightarrow V$ is a given function. If $F:(a, b) \rightarrow V$ is a solution to the functional inequality on $V$, then $F$ is strongly convex on $(a, b)$, and

$$
F(t)-F(a+\varepsilon)=\int_{a+\varepsilon}^{t} G(s) d s, \text { where } a<a+\varepsilon<t<b .
$$

The integral on the right is of Riemann type and convergent relative to the orderconvergence. Suppose further that $V$ is a Banach lattice and $G$ is nonnegative on $(a, b)$ in the sense that $\theta \ll G(s)$ for all $s$ in $(a, b)$; then the Bochner integral (B) $\int_{a+\varepsilon}^{t} G(s) d s$ converges under the norm on $V$ and

$$
\text { (B) } \int_{a+\varepsilon}^{t} G(s) d s=F(t)-F(a+\varepsilon) .
$$

Proof. Since $F$ satisfies the functional inequality (1), we have:

$$
\begin{aligned}
& F(s)+t G(s) \ll F(s+t), \quad \text { whenever } s \text { and } s+t \text { in }(a, b) . \\
& F(s)-t G(s) \ll F(s-t), \quad \text { whenever } s \text { and } s-t \text { in }(a, b) .
\end{aligned}
$$

From (2) and (3) it follows that

$$
2 F(s) \ll F(s+t)+F(s-t), \quad \text { whenever } s+t \text { and } s-t \text { in }(a, b) .
$$

This is equivalent to $F\left(\frac{1}{2} s+\frac{1}{2} t\right) \ll \frac{1}{2} F(s)+\frac{1}{2} F(t)$, whenever $s$ and $t$ are in $(a, b)$. Thus $F$ is strongly convex on $(a, b)$.

The function $G$ is isotone (nondecreasing) on $(a, b)$, because the following two inequalities hold for positive small $\delta: F(s-\delta)+\delta G(s-\delta) \ll F(s)$ and $F(s)-\delta G(s) \ll F(s-\delta)$.

Since $G$ is monotone, it is natural to use step functions in proving that $G$ is Riemann integrable over $[a+\varepsilon, t]$ where $0<\varepsilon$ and $a+\varepsilon \leqslant t<b$. In the process of doing so, it can be assumed, without loss of generality, that $a+\varepsilon=0$ and $t=1$. Let simple functions $\Phi_{n}$ and $\Psi_{n}$ be constructed by

$$
\Phi_{n}=\sum_{i=0}^{n-1} G\left(\frac{i}{n}\right) \chi\left[\frac{i}{n}, \frac{i+1}{n}\right], \quad \Psi_{n}=\sum_{i=1}^{n} G\left(\frac{i}{n}\right) \chi\left[\frac{i-1}{n}, \frac{i}{n}\right],
$$

where $\chi[i / n,(i+1) / n]$ is the characteristic function on the interval $[i / n$, $(i+1) / n]$.

From the inequalities $\delta G(s) \ll F(s+\delta)-F(s)$ and $-\delta G(s) \ll F(s-\delta)$

$-F(s)$ it follows that 


$$
\frac{1}{n} G\left(\frac{i}{n}\right) \ll F\left(\frac{i+1}{n}\right)-F\left(\frac{i}{n}\right) \ll \frac{1}{n} G\left(\frac{i+1}{n}\right) \text { for } i=0, \ldots, n-1 .
$$

Therefore,

$$
\begin{aligned}
\int_{0}^{1} \Phi_{n} d s & \stackrel{\text { def }}{=} \frac{1}{n} \sum_{i=0}^{n-1} G\left(\frac{i}{n}\right) \ll \sum_{i=0}^{n-1}\left[F\left(\frac{i+1}{n}\right)-F\left(\frac{i}{n}\right)\right] \\
& =\sum_{i=1}^{n}\left[F\left(\frac{i}{n}\right)-F\left(\frac{i-1}{n}\right)\right] \\
& =F(1)-F(0) \ll \sum_{i=1}^{n} \frac{1}{n} G\left(\frac{1}{n}\right) \stackrel{\text { def }}{=} \int_{0}^{1} \Psi_{n} d s .
\end{aligned}
$$

Thus,

$$
\int_{0}^{1} \Phi_{n} d s \ll F(1)-F(0) \ll \int_{0}^{1} \Psi_{n} d s
$$

Furthermore,

(5) $\int_{0}^{1} \Psi_{n} d s-\int_{0}^{1} \Phi_{n} d s=\frac{1}{n} \sum_{i=1}^{n}\left[G\left(\frac{i}{n}\right)-G\left(\frac{i-1}{n}\right)\right]=\frac{1}{n} G(1)-\frac{1}{n} G(0)$.

From (4) it follows that

$$
\sup \int_{0}^{1} \Phi_{n} d s \ll F(1)-F(0) \ll \inf \int_{0}^{1} \Psi_{n} d s .
$$

From (5) it follows that

$$
\inf \int_{0}^{1} \Psi_{n} d s-\sup \int_{0}^{1} \Phi_{n} d s \ll \inf \frac{1}{n}[G(1)-G(0)]=\theta .
$$

Combining (6) and (7) yields

$$
\sup \int_{0}^{1} \Phi_{n} d s=\inf \int_{0}^{1} \Psi_{n} d s=F(1)-F(0) .
$$

Thus with respect to the order convergence, $G$ is Riemann integrable and

$$
\int_{a+\varepsilon}^{t} G(s) d s=\inf \int_{a+\varepsilon}^{t} \Psi_{n} d s=\sup \int_{a+\varepsilon}^{t} \Phi_{n} d s=F(t)-F(a+\varepsilon) .
$$

Under the additional assumption that $V$ is a Banach lattice, the function $G$ is strongly Lebesgue measurable on $[a+\varepsilon, t]$, since the sequence of simple functions $\left\{\Phi_{n}\right\}$ converges strongly to $G$ due to the construction of $\Phi_{n}$. The realvalued function $\|G(s)\|$ is nondecreasing on $[a+\varepsilon, t]$, and, therefore, Lebesgue integrable, since the vector-valued function $G$ with $\theta \ll G(s)$ is isotone in the Banach lattice $V$. The strong measurability of $G$ and the integrability of $\|G(s)\|$ imply that the Bochner integral $(B) \int_{a+\varepsilon}^{t} G(s) d s$ exists [7, p. 133]. To establish (B) $\int_{a+\varepsilon}^{t} G(s) d s=F(t)-F(a+\varepsilon)$, it suffices to prove that, for each bounded linear functional $q$ in $V^{*}$,

$$
\int_{a+\varepsilon}^{t} q[G(s)] d s=q[F(t)]-q[F(a+\varepsilon)] .
$$


This can be shown in two steps. First, use the well-known Riesz Decomposition Theorem [8, p. 68] to obtain $q=q^{+}-q^{-}$, where $q^{+}$and $q^{-}$are positive linear functionals on $V$. Secondly, to the functional inequality $q^{+}[F(s)]$ $+t q^{+}[G(s)] \leqslant q^{+}[F(s+t)]$ apply the step-function method, just as has been done to (1), and infer that

$$
\int_{a+\varepsilon}^{t} q^{+}[G(s)] d s=q^{+}[F(t)]-q^{+}[F(a+\varepsilon)] .
$$

Similarly,

$$
\int_{a+\varepsilon}^{t} q^{-}[G(s)] d s=q^{-}[F(t)]-q^{-}[F(a+\varepsilon)]
$$

Thus,

$$
\begin{aligned}
\int_{a+\varepsilon}^{t} q[G(s)] d s & =\int_{a+\varepsilon}^{t}\left(q^{+}-q^{-}\right)[G(s)] d s \\
& =\left(q^{+}-q^{-}\right)[F(t)]-\left(q^{+}-q^{-}\right)[F(a+\varepsilon)] \\
& =q[F(t)]-q[F(a+\varepsilon)] .
\end{aligned}
$$

From (8) and the Hahn-Banach Theorem, it follows that $(B) \int_{a+\varepsilon}^{t} G(s) d s$ $=F(t)-F(a+\varepsilon)$. This completes the proof of the theorem.

ACKNOWLEDGEMENT. The author wishes to thank the referee for suggesting several improvements in this paper, particularly the formulation of the Lemma.

\section{REFERENCES}

1. G. Birkhoff, Lattice theory, 3rd ed., Amer. Math. Soc. Colloq. Publ., vol. 25, Amer. Math. Soc., Providence, R. I., 1967. MR 37 \#2638.

2. A. Wilansky, Functional analysis, Blaisdell, New York, 1964. MR 30 \#25.

3. B. Z. Vulih, Introduction to the theory of partially ordered spaces, Fizmatgiz, Moscow, 1961; English transl., Wolters-Noordhoff, Groningen, 1967. MR 24 \# A3494; 37 \#121.

4. I. Hsu and R. G. Kuller, Convexity of vector-valued functions, Proc. Amer. Math. Soc. 46 (1974), 363-366.

5. G. H. Hardy, J. E. Littlewood and G. Pólya, Inequalities, 2nd ed., Cambridge Univ. Press, London and New York, 1952. MR 13, 727.

6. A. W. Roberts and D. E. Varberg, Convex functions, Academic Press, New York-London, 1973.

7. K. Yosida, Functional analysis, 3rd ed., Springer-Verlag, Berlin and New York, 1971.

8. G. J. O. Jameson, Ordered linear spaces, Lecture Notes in Math., no. 141, Springer-Verlag, Berlin and New York, 1970.

Department of Mathematics, St. Olaf College, Northfield, Minnesota 55057 